\title{
Monte Carlo study of coherent scattering effects of low-energy charged particle transport in Percus-Yevick liquids
}

\author{
W. J. Tattersall, ${ }^{1,2}$ D. G. Cocks, ${ }^{2}$ G. J. Boyle, ${ }^{2}$ S. J. Buckman, ${ }^{1,3}$ and R. D. White ${ }^{2}$ \\ ${ }^{1}$ Research School of Physics and Engineering, The Australian National University, Canberra, ACT 0200, Australia \\ ${ }^{2}$ College of Science, Technology and Engineering, James Cook University, Townsville 4810, Australia \\ ${ }^{3}$ Institute of Mathematical Sciences, University of Malaya, 50603 Kuala Lumpur, Malaysia
}

(Received 30 January 2015; published 16 April 2015)

\begin{abstract}
We generalize a simple Monte Carlo (MC) model for dilute gases to consider the transport behavior of positrons and electrons in Percus-Yevick model liquids under highly nonequilibrium conditions, accounting rigorously for coherent scattering processes. The procedure extends an existing technique [Wojcik and Tachiya, Chem. Phys. Lett. 363, 381 (2002)], using the static structure factor to account for the altered anisotropy of coherent scattering in structured material. We identify the effects of the approximation used in the original method, and we develop a modified method that does not require that approximation. We also present an enhanced MC technique that has been designed to improve the accuracy and flexibility of simulations in spatially varying electric fields. All of the results are found to be in excellent agreement with an independent multiterm Boltzmann equation solution, providing benchmarks for future transport models in liquids and structured systems.
\end{abstract}

DOI: 10.1103/PhysRevE.91.043304

PACS number(s): 05.10.-a, 05.20.Dd, 34.80.Bm, 61.20.Ne

\section{INTRODUCTION}

The precise behavior of electrons and positrons traveling through matter is of vital importance in many new and established technologies. Applications such as solar cells [1], radiation dosimetry [2], material pore-size classification [3], and positron emission tomography [4] all require an understanding of the fundamental physical processes involved, including an accurate knowledge of energy deposition, macroscopic behaviors, and loss rates.

Although the behavior of high-energy particles can be simulated quite accurately with condensed history techniques [5], at low energies it is important to model the individual interactions of particles colliding with the background material, and thereby monitor discrete energy losses and processes that change the number of particles in the system. In the systems that we are investigating, the number density of the charged particles is low enough that the Debye wavelength greatly exceeds the dimensions of the system, which is known as the "swarm" limit of an ionized gas [6].

One successful approach to modeling such systems is by solving the Boltzmann equation [7], which is an equation of continuity in phase space. Often this approach is limited to idealized geometries, due to complexities in the numerical solution and application of boundary conditions. However, many real-world systems are too complex for such an approach to be effective, and in any case, alternative methods should ideally be used for verification.

The preeminent alternative is to use Monte Carlo simulations, which have been widely employed for such purposes [8-12] ever since computers have been powerful enough to implement them [13]. Monte Carlo simulations are very flexible, and they can easily include features from systems that are quite difficult to model in any other manner, such as interfacial effects, secondary particles, and inhomogeneous media.

Accurate simulations of condensed systems must include the effects of coherent scattering, where the incoming electrons and positrons interact with many particles of the system at once. This can occur when the de Broglie wavelength of the low-energy electrons and positrons is longer than the mean distance between molecules of the condensed matter [14]. It is common to ignore these effects in Monte Carlo simulations of liquids $[15,16]$, perhaps because they are usually insignificant for electrons and positrons with energies greater than $\sim 10-20 \mathrm{eV}$. However, to accurately treat particle transport at low energies, we must include these collective effects, usually by way of the medium's dynamic structure factor $S(\Delta \mathbf{k}, \omega)$ [14], which contains information about the medium's characteristic allowed transfers of momentum $\hbar \Delta \mathbf{k}$ and energy $\hbar \omega$. The dynamic structure is exactly what is measured by coherent neutron scattering experiments such as [17].

From the perspective of solid-state physics, the dynamic structure factor is directly related to the complex permittivity function, $\varepsilon(\Delta k, \omega)$ [see Eq. (14) in [18]]. It is common to treat such systems in a phenomenological manner, where all scattering processes are combined into an effective energyloss function, by taking the limit of $\Delta k \rightarrow 0$, known as the optical limit. A Monte Carlo technique can use the energy-loss function to model experiments and perfectly emulate the energy transfer rates of experiment (see, for example, [19] and [20]), without assuming any knowledge of the underlying processes. However, this approach does not attempt to provide a precise microscopic view of the collisional processes, which means it is not possible to account for process-dependent anisotropic scattering or momentum transfer without additional modifications to the scattering procedure.

The present study will describe a Monte Carlo implementation that models structured matter using a static structure factor, $S(\Delta \mathbf{k})$, which is an integrated form of the dynamic structure factor. We first use a technique described by Wojcik and Tachiya [21] to incorporate the static structure factor into our Monte Carlo model, but we assert that this method is only accurate for a certain subset of cases. We subsequently extend 
this technique to overcome its limitations. The Percus-Yevick static structure factor is a simple analytic static structure factor [22] that can be used as a benchmark to verify the accuracy of our simulation. We have performed simulations of a number of Percus-Yevick systems at a range of reduced electric-field strengths, and we compare our results with those obtained by solving the Boltzmann equation detailed in [7].

We begin this study with a discussion of the Boltzmann equation approach to coherent scattering. We follow this with a brief description of typical Monte Carlo collision mechanics for elastic processes in Sec. II A. We then use the Boltzmann equation coherent scattering rates to derive a set of modified cross sections in Sec. II B, and we identify the approximation made in Wojcik and Tachiya's original method [21]. In Sec. II C, we describe a method that we have developed for performing simulations in spatially varying electric fields. The model system that we are studying is extensively described in Sec. III, and finally we present our results in Sec. IV, including comparisons with the results from both our implementation of the method of Wojcik and Tachiya, as well as an independent Boltzmann equation solution.

\section{THEORY}

\section{A. Coherent scattering}

Designing simulations of swarm transport in liquids and dense gases presents additional challenges compared to the ideal-gas case. Because the interparticle spacing of the neutral particles is often less than the de Broglie wavelength of the swarm particles, the swarm particles must often interact with several neutral particles at the same time, which means that any spatial or temporal correlations between said particles will have an effect on scattering events. The Cohen-Lekner theory of electron transport [23] describes these effects in terms of two rates of transfer-momentum and energy - that occur independently.

Cohen and Lekner express the electron distribution function in a basis of spherical harmonics. They then modify the standard Boltzmann collision integral to include the dynamic structure factor $S(\Delta \mathbf{k}, \omega)$, as motivated by van Hove's definition of the ensemble cross section [24], and then they show that when the necessary integrals have been performed, the dependence is only upon the static structure factor.

Upon solving the equations for the time evolution of the distribution function, they ascribe a physical meaning to two of the mean free path lengths that appear in the collision integral expansion. The first fully determines the rate of energy transferred from the swarm particles. It is independent of the structure of the medium, and it is given by the mean free path corresponding to single-particle elastic scattering:

$$
\begin{aligned}
\Lambda_{0} & =\left(n_{0} \sigma_{m}\right)^{-1} \\
& =\left(n_{0} 2 \pi \int_{0}^{\pi} d \chi \sin \chi(1-\cos \chi) \sigma_{\mathrm{sp}}(\epsilon, \chi)\right)^{-1},
\end{aligned}
$$

where $n_{0}$ is the number density of the neutral molecules, $\sigma_{\mathrm{sp}}(\epsilon, \chi)$ is the angle-differential elastic cross section for scattering with a single particle (also known as the binary or gas-phase cross section), and $\sigma_{m}$ is the usual definition of the momentum transfer cross section in the absence of coherent effects. Throughout the present work, $\epsilon$ refers to the relative energy in the center-of-mass frame during a collision, and $\chi$ represents the angle through which the relative velocity is changed.

The second mean free path partly includes the effect of the medium and contains all information about the rate at which momentum is transferred:

$$
\begin{aligned}
\Lambda_{1}=\left(n_{0} \tilde{\sigma}_{m}\right)^{-1}= & \left(n_{0} 2 \pi \int_{0}^{\pi} d \chi \sin \chi(1-\cos \chi)\right. \\
& \left.\times \sigma_{\mathrm{sp}}(\epsilon, \chi) S(\Delta \mathbf{k})\right)^{-1}
\end{aligned}
$$

where $S(\Delta \mathbf{k})$ is the static structure factor as a function of the momentum transferred and $\tilde{\sigma}_{m}$ represents a structure modification of the momentum transfer cross section.

In a recent paper [25], the explicit rates of energy and momentum transfer were calculated with the inclusion of structure in the Boltzmann equation. The components of this transfer due to the collision term, in the case of zero temperature, are

$$
\begin{aligned}
\left.\frac{d}{d t}\langle n m \boldsymbol{v}\rangle\right|_{\text {coll }} & =-\left\langle n_{0} v \tilde{\sigma}_{m}(v) m \boldsymbol{v}\right\rangle+O(\omega)+O\left(\frac{m}{m_{0}}\right) \\
& =-\left\langle v \Lambda_{1}^{-1}(v) m \boldsymbol{v}\right\rangle
\end{aligned}
$$

and

$$
\begin{aligned}
\left.\frac{d}{d t}\langle n \epsilon\rangle\right|_{\text {coll }} & =-2 \frac{m}{m_{0}}\left\langle n_{0} v \sigma_{m}(v) \epsilon\right\rangle+O\left(\omega^{2}\right)+O\left[\left(\frac{m}{m_{0}}\right)^{2}\right] \\
& =-2 \frac{m}{m_{0}}\left\langle v \Lambda_{0}^{-1}(v) \epsilon\right\rangle
\end{aligned}
$$

where \langle\rangle represents averaging over velocity space and $n$ is the number density of the charged particles.

Note that these representative mean free paths should be considered independently, and should only be thought of as an average rate of transfer of the relevant quantity, rather than as a prescription for separate collision events. We define the ratio $\Gamma(\epsilon) \equiv \Lambda_{0} / \Lambda_{1}=\tilde{\sigma}_{m} / \sigma_{m}$. In the dilute gas case, $\Gamma(\epsilon)=1$, because the static structure factor of a dilute gas is unity for all momentum transfers. However, in a structured medium such as a dense gas or a liquid, the ratio can deviate markedly from unity. If $\Gamma(\epsilon)<1$, there is noticeably less momentum transfer than in the single-particle scattering case, which can be interpreted as a preference toward forward scattering events. In the opposite case of $\Gamma(\epsilon)>1$, more momentum transfer occurs, which causes the particle to change direction without losing as much energy as it would in the single-particle scattering.

In the case of an isotropic single-particle elastic cross section, $\sigma_{\mathrm{sp}}(\epsilon, \chi)=\frac{1}{4 \pi} \sigma_{\mathrm{sp}}(\epsilon)$, as in the model described in Sec. III, and the ratio $\Gamma(\epsilon)$ reduces to

$$
\begin{aligned}
\Gamma(\epsilon)=\frac{\Lambda_{0}}{\Lambda_{1}}= & \frac{1}{2} \int_{0}^{\pi} d \chi \sin \chi(1-\cos \chi) \\
& \times S\left(\frac{2(2 m \epsilon)^{1 / 2}}{\hbar} \sin \left(\frac{\chi}{2}\right)\right),
\end{aligned}
$$


where we have assumed the static structure factor depends only on the magnitude of $\Delta \mathbf{k}$, and $|\hbar \Delta \mathbf{k}|=\hbar \Delta k \approx 2 \sqrt{2 m \epsilon} \sin \frac{\chi}{2}$ in the limit of a small mass ratio $m / m_{0}$. Throughout the present work, $m$ refers to the mass of each charged particle, and $m_{0}$ is the mass of each neutral molecule. This form of $\Gamma(\epsilon)$ is sometimes called the angle-integrated static structure factor, $\bar{S}(\epsilon)$, and it is this form of the structure factor that is used in several previous works [7,21].

In the case of dilute gases, where $S(\Delta \mathbf{k})=1$, the energy and momentum transfer rates converge, yielding the single-scattering model in which every energy transfer is accompanied by a momentum transfer. When the transfer rates differ, however, this theory is not directly applicable to Monte Carlo modeling because it does not give a microscopic description of how much energy and momentum is transferred in each collision between swarm particles and neutral particles.

\section{B. Sampling coherent scattering in Monte Carlo simulations}

Our Monte Carlo simulations are built around sampling sets of scattering cross sections, $\sigma$, that define the probabilities of all interactions between the charged particles and the medium. Each cross section represents a single type of scattering process, for example elastic, direct ionization, or a particular electronic excitation of the neutral. They usually depend upon the relative speed of the charged particle during a collision, and on the scattering angle $\chi$. In the case of a cold background medium, the collision frequency is simply given by

$$
v=n_{0} v \sigma_{\mathrm{tot}}(v),
$$

where $v$ is the speed of the charged particle and $\sigma_{\text {tot }}(v)$ is the integrated sum of all differential cross sections at that speed [26]. This quantity is used to stochastically sample the time between each collision (further described in Sec. II C). When a collision is simulated, a specific cross section is randomly selected according to the relative probabilities of the available cross sections [27]. In the case of single-scattering collisions with independent gas molecules, the amount of energy and momentum transferred is fully determined by the initial energy and the scattering angles.

For structured materials, an approximate theory has been developed by Wojcik and Tachiya [21], who propose a

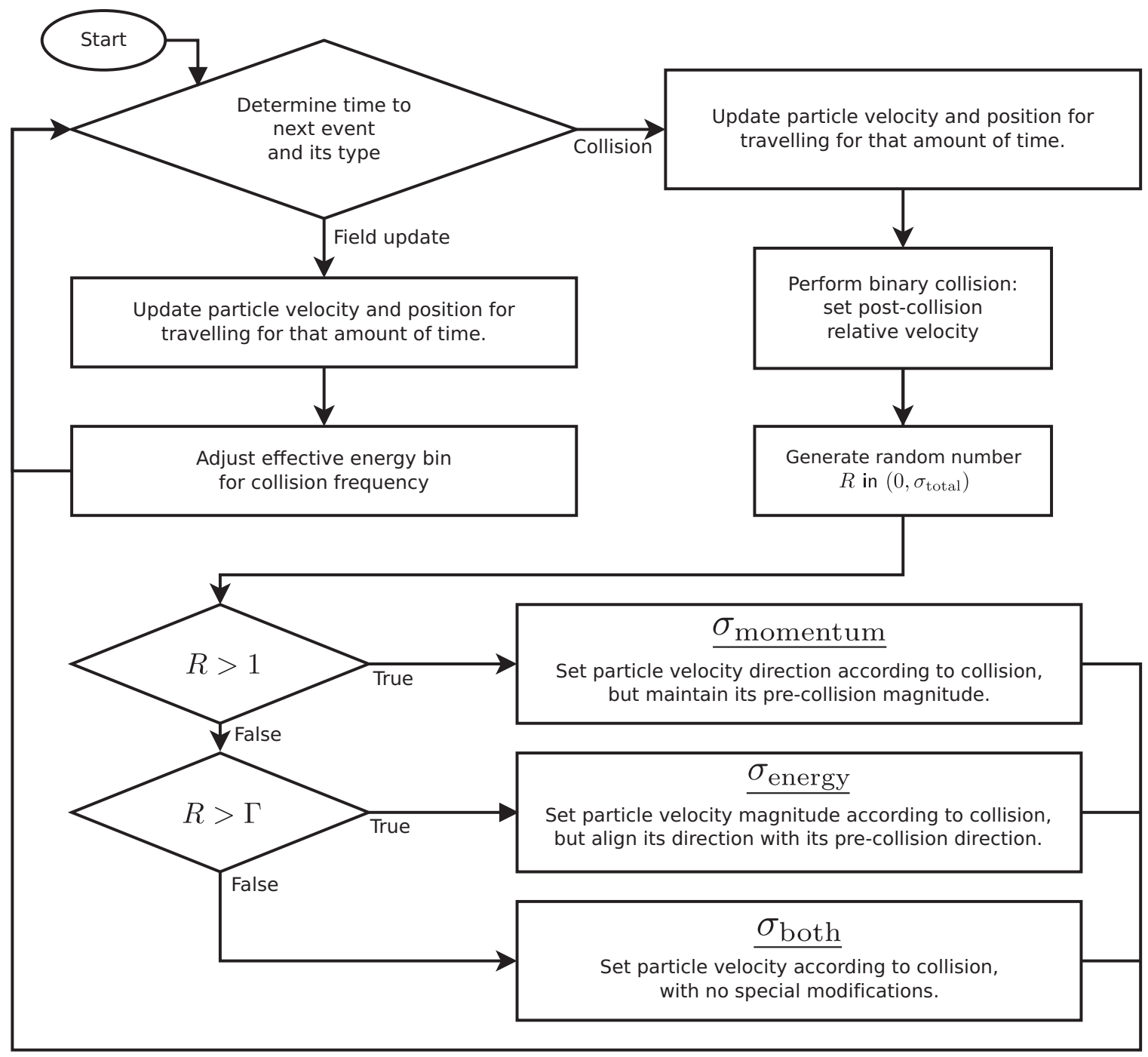

FIG. 1. Flowchart detailing how electric fields and coherent scattering are implemented in the SSMC code. 


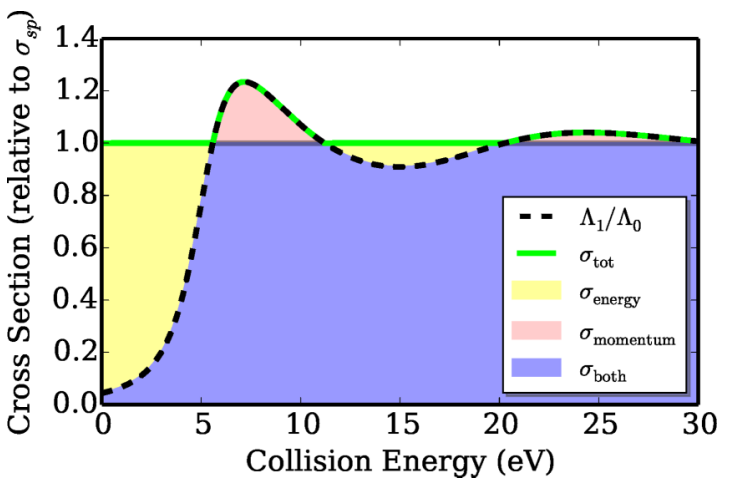

FIG. 2. (Color online) Schematic diagram of the various elastic cross sections used in simulating a Percus-Yevick liquid $(\phi=0.4)$. All quantities are given relative to the elastic cross section for a single particle. Note that $\sigma_{\mathrm{tot}} \geqslant \sigma_{\mathrm{sp}}$.

mechanistic model of electron transport in rare-gas liquids. In what follows, we have extended this model to be more generally applicable to other systems, highlighting the approximations and associated errors of Wojcik and Tachiya's model.

The presence of structure requires the introduction of additional microscopic processes that, at a macroscopic level, produce the same rate of energy and of momentum transfer as in the Boltzmann equation formalism detailed in Sec. II A. We choose to do this by separating the original, single-particle elastic cross section into three different processes depending on the ratio $\Gamma(\epsilon)$, as illustrated in Fig. 2. These processes have cross sections, labeled by which quantities are affected in the collision: $\sigma_{\text {both }}, \sigma_{\text {momentum }}$, and $\sigma_{\text {energy }}$. The result of a collision from process $\sigma_{\text {both }}$ is identical to that of a regular single-particle scattering collision. For $\sigma_{\text {energy, }}$ we start with a regular single-particle scattering collision, but we set the postcollision direction of motion for the particle to be unchanged. This has the effect of transferring a minimal amount of momentum while maintaining the same energy transfer as in $\sigma_{\text {both}}$. For $\sigma_{\text {momentum, }}$, we perform a regular single-particle scattering collision, but we scale the postcollision particle speed to be equal to that before the collision. This results in exactly zero transfer of energy, but some change of vector momentum.

The path lengths $\Lambda_{0}$ and $\Lambda_{1}$ in Sec. II A correspond to transfer rates of $v_{m}=v n_{0} \sigma_{m}=v / \Lambda_{0}$ and $\tilde{v}_{m}=v n_{0} \tilde{\sigma}_{m}=$ $v / \Lambda_{1}$ for energy and momentum, respectively, where $v$ is the speed of the charged particle. To achieve these rates, we combine the cross sections in various ratios depending on the value of $\Gamma(\epsilon)=\Lambda_{0} / \Lambda_{1}$. If $\Gamma(\epsilon)<1$, we wish to decrease the rate of momentum transfer while maintaining energy transfer, and so we choose $\sigma_{\text {both }}^{\Gamma<1}=\Gamma(\epsilon) \sigma_{\text {sp }}, \sigma_{\text {energy }}^{\Gamma<1}=[1-\Gamma(\epsilon)] \sigma_{\text {sp }}$, and $\sigma_{\text {momentum }}^{\Gamma<1}=0$. In the opposite case, $\Gamma(\epsilon)>1$, we achieve an increased rate of momentum transfer by setting $\sigma_{\text {both }}^{\Gamma>1}=\sigma_{\mathrm{sp}}$, $\sigma_{\text {momentum }}^{\Gamma>1}=[\Gamma(\epsilon)-1] \sigma_{\mathrm{sp}}$, and $\sigma_{\text {energy }}^{\Gamma>1}=0$. This gives a total elastic cross section of $\sigma_{\text {tot }}=\max (1, \Gamma(\epsilon)) \sigma_{\mathrm{sp}}$. The complete Monte Carlo procedure, which we refer to as the static structure Monte Carlo (SSMC) method, is shown as a flowchart in Fig. 1.

We emphasize that these collision processes do not individually represent multiple scattering interactions between the electrons and the medium-in particular, the momentum and energy-only collisions would require rather contrived circumstances to achieve momentum energy conservation between the electron and the medium. Instead, the additional collision processes are designed to reproduce the rates of energy and momentum transfer in Eqs. (1) and (2) when considered as the average of a series of selected collisions. However, it is not obvious that our construction of the microscopic processes achieves this goal. In Appendix A, we show that such a sampling process involving these cross sections does indeed satisfy these requirements, to within the order of the mass ratio $\mathrm{m} / \mathrm{m}_{0}$ as mentioned earlier. These differences are small enough that they are unlikely to effect electron-atom simulations, though they may be significant in systems where ions serve as the charged particles.

Wojcik and Tachiya [21] studied liquid argon according to the method described above, but their mechanistic model, hereafter referred to as the WT method, effectively capped the value of $\Gamma(\epsilon)$ such that it never exceeded unity. This meant that their total collision frequency was unaltered from the single-particle scattering case, and simulation of the particles in the energy regions where $\Gamma(\epsilon)$ exceeded 1 could only be considered approximately accurate. The difference between the SSMC and WT methods [21] is shown in Fig. 2, where the regions labeled $\sigma_{\text {momentum }}$ are absent in their model, and the total cross section is modified accordingly, so that it is simply $\sigma_{\text {sp. }}$. For the aforementioned study of liquid argon, such modifications were only required in a small energy range for the structure factor that they employed. One of the purposes of the present study is to determine how this approximation affects the results for a benchmark Percus-Yevick model, where the approximation is more significant.

\section{Precise treatment of electric fields in Monte Carlo simulations}

Electric fields present a particular challenge for this style of Monte Carlo simulation. As the collision frequency $v$ of a given charged particle is dependent on its energy $\epsilon$ [see Eq. (6)], the time between collisions $\tau$ can be altered by the change of energy of the particle due to the electric field, even as it is undergoing transport between collisions. Mathematically, the probability of a time between collisions greater than $\tau$ can be expressed as [13]

$$
P(\tau)=\exp \left(-\int_{0}^{\tau} v(\epsilon(t)) d t\right),
$$

where the charged particle's energy is time-dependent due to the particle's passage through an electric field. Explicitly performing this integral for every collision would be very computationally expensive, given that the changes in $\epsilon$ will depend on the velocity at which the particle is traveling and, for nonuniform electric fields, the position of the charged particle as well.

One popular approach uses the method of "null collisions" [13], where the collision frequency is calculated based on the maximum collision frequency $v_{0}$ that the particle is likely to be able to reach during its transport. Using this constant 
collision frequency, Eq. (7) can be solved by equating $P(\tau)$ with a uniformly distributed random number $R$, in which case

$$
\tau=-v_{0}^{-1} \ln R .
$$

When the collision occurs, a second random number is generated that is used to account for this overestimation by allowing the charged particle to undergo "null" collisions, where no exchange of energy or momentum occurs. This procedure suffers from a requirement to "backtrack" if the assumed collision frequency is too low, where it must then make a second assumption with a higher collision frequency. It is therefore important to minimize the number of null collisions and backtracks to optimize the simulation speed, and more modern simulations [28] have been designed with this in mind. The null collision method effectively amounts to a form of rejection method for sampling from $P(\tau)$, which means that potentially many random numbers are generated for each valid collision.

The simulation presented here uses an alternative approach. The cross sections are specified as a function of energy, but they are assumed to be constant within energy bins of width $\delta \epsilon$. These energy bins can be made arbitrarily small, so there is no loss of accuracy provided that we are careful to test that the results are independent of the bin width. However, this means that it is sufficient to recalculate $\tau$ only when the energy of the particle changes from one bin to another, so until this occurs, the collision frequency in Eq. (8) remains constant. We therefore design the simulation so that particles can undergo two types of interactions. Collisions with neutral particles are described above, and they are governed by $i$, the energy bin of the particle. The second type of interaction occurs when the energy bin is judged to have changed due to the effect of the electric field. In such an interaction, the only parameter that changes is $i$, which either increases or decreases by 1 , triggering a recalculation of the time until the next neutral particle interaction. The time until the change, $t$, is determined by analysis of the particle's current velocity and the (constant) acceleration that it is experiencing due to the electric field. This is given by the smallest real, positive solution to the following equation for the kinetic energy:

$$
\frac{1}{2} m\left[\mathbf{v}_{\mathbf{0}}+(\mathbf{a} t)\right]^{2}=\epsilon_{i} \pm \frac{1}{2} \delta \epsilon .
$$

Recalculating the time until collision $\tau$ does not require the use of another random number. Recalling Eq. (8), we now have additional terms for each change in energy bin:

$$
\begin{aligned}
\tau= & t_{0}+t_{1}+\cdots+t_{n} \\
& -v_{n}^{-1}\left[\ln (R)-v_{0} t_{0}-v_{1} t_{1}-\cdots-v_{n-1} t_{n-1}\right],
\end{aligned}
$$

where each $t_{i}$ is determined by the time required for the particle to change energy from one bin to the next, and each $v_{i}$ represents the corresponding collision frequency for each energy bin at that time. This equation reduces to Eq. (8) in the case of constant $v_{n}$ or zero $t_{i}$. In practice, the simulation maintains a running measure of the remaining "collision probability" for each particle. This is the dimensionless quantity in square brackets in Eq. (10) that is

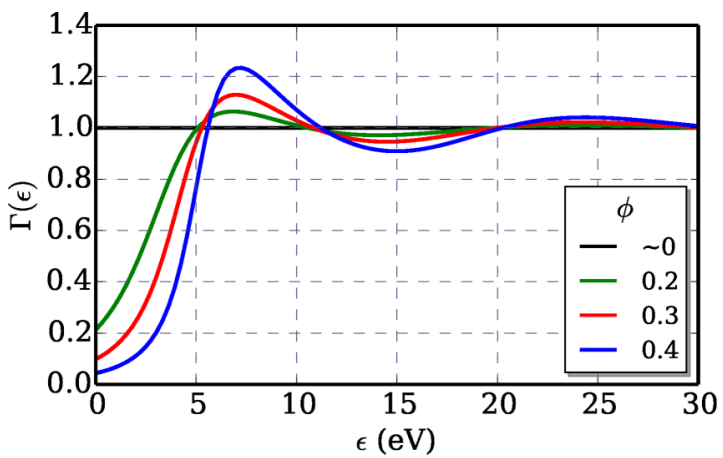

FIG. 3. (Color online) Angle-integrated Percus-Yevick structure factors, from Eqs. (11) and (5), as a function of particle energy and volume fraction.

divided by the current $v$ to calculate the time until the next collision.

\section{BENCHMARK SYSTEM FOR MODELING COHERENT SCATTERING}

\section{A. Percus-Yevick hard-sphere model}

To demonstrate and benchmark the proposed simulation procedure and code, we apply it to a simple model system that requires a correct treatment of structured media. One such model, frequently used in the literature, is that of a structure for hard-sphere potentials obtained by applying the Percus-Yevick approximation as a closure to the Ornstein-Zernike equation, which yields a pair-correlation function $[29,30]$, which in turn can be transformed into a static structure factor via a Fourier transform and directly used in our simulation. We use the Verlet and Weiss [22] structure factor, which includes some corrections to better emulate the structure of a real liquid:

$$
\begin{aligned}
S(\Delta k)= & \left(1+\frac{24 \eta}{\Delta k^{2}}\left[\frac{2}{\Delta k^{2}} \frac{12 \zeta}{\Delta k^{2}-\beta}\right.\right. \\
& +\frac{\sin (\Delta k)}{\Delta k}\left(\alpha+2 \beta+4 \zeta-\frac{24 \zeta}{\Delta k^{2}}\right) \\
& \left.\left.+\frac{2 \cos (\Delta k)}{\Delta k^{2}}\left(\beta+6 \zeta-\frac{12 \zeta}{\Delta k^{2}}\right)-\alpha-\beta-\zeta\right]\right)^{-1},
\end{aligned}
$$

where $\eta=\phi-\frac{\phi^{2}}{16}, \alpha=\frac{(1+2 \eta)^{2}}{(1-\eta)^{4}}, \beta=\frac{-6 \eta\left(1+\frac{\eta}{2}\right)^{2}}{(1-\eta)^{4}}$, and $\zeta=\frac{\eta \alpha}{2}$. This includes a packing density parameter, $\phi$, which specifies how closely the hard spheres are packed. It can be written in terms of the hard-sphere radius $r$ and the neutral number density $n_{0}$ as $\phi=\frac{4}{3} \pi r^{3} n_{0}$. This structure factor depends only on the magnitude of the momentum exchange during a collision.

We have modeled systems with a range of densities, from $\phi \approx 0$, which approximates a dilute gas, to $\phi=0.4$, which states that $40 \%$ of the volume is excluded by the hard-sphere potentials of the neutral molecules. The angle-integrated forms of each of these structure factors, as described in Eq. (5), are shown in Fig. 3. 


\section{B. System parameters}

Our Monte Carlo codes use an event-by-event model in which every collision is considered independently. This allows for a great deal of flexibility in the specification of scattering mechanics, without any of the approximations used by "condensed history" simulations (see, e.g., [31]). In addition, the swarm approximation - that all transport particles are independent-allows the simulation to be run in parallel. This makes it ideal for scheduled multiprocessor batch jobs, where execution is not necessarily simultaneous or even sequential.

We have calculated a number of transport coefficients for comparison with other models. The meaning and derivation of all of these coefficients are described in [7] and [32], but a short summary is given here. All coefficients are measured as a discrete function of time step $t_{i}$. During simulation, if a particle's history crosses a time step, its properties (e.g., position, velocity, and position squared) at that time are sampled. We choose the $z$ axis to be aligned with the electric field, since it is the only element of the system that has a preferred direction. Each property is added to a separate running total for each time step, and after the simulation is complete, the totals are divided by the (in general) timedependent total of the number of particles. This results in the average of a property over all particles, as a function of time. While the transport coefficients are generally time-varying, for the systems considered in this study all transport coefficients eventually reach a hydrodynamic equilibrium after sufficient time has passed. Afterward, they merely fluctuate in a small statistical range about the reported equilibrium value.

Depending on the required transport coefficients, different properties of the charged particles must be recorded. In the following table, each definition [32] is of the named property at one point in time:

\begin{tabular}{ll} 
Transport coefficient & Definition \\
\hline Mean energy & $\bar{\epsilon}=\langle\epsilon\rangle$ \\
Bulk drift velocity & $W=\frac{d}{d t}\left\langle r_{z}\right\rangle$ \\
Bulk longitudinal diffusion & $D_{L}=\frac{d}{d t}\left(\left\langle r_{z}^{2}\right\rangle-\left\langle r_{z}\right\rangle^{2}\right)$ \\
Bulk transverse diffusion & $D_{T}=\frac{1}{2} \sum_{i=x, y} \frac{d}{d t}\left(\left\langle r_{i}^{2}\right\rangle-\left\langle r_{i}\right\rangle^{2}\right)$
\end{tabular}

Angular brackets denote an average over all particles, $r_{i}$ represents the appropriate Cartesian coordinate of the position of the particle, and $\epsilon$ represents the energy of the particle.

In principle, there are two types of transport coefficients, known as "flux" and "bulk," which approximately correspond to per-particle averages and system averages, respectively [32]. However, because our model contains no nonconservative collision processes, the "flux" and "bulk" quantities should be identical, provided enough time samples are taken. We have chosen to measure "bulk" quantities where possible, because this implicitly averages over changes in velocity between time steps, whereas measuring the flux drift velocity and diffusion requires sampling instantaneous values at discrete time values. For the same number of time steps, without any explicit time-averaging of velocity, the bulk quantities have far less statistical error.
Two ranges of reduced field strengths were used. An approximately logarithmic spacing of electric field strengths from 0.001 to $100 \mathrm{Td}$ provides a broad picture of the resulting behaviors, while a linear spacing of field strengths from 2 to 12 Td provides detail in the range in which we expect the two Monte Carlo methods to disagree most strongly.

We employ the cold-gas limit, in which the neutrals are considered to be at rest. The present method does not accurately support nonzero neutral temperatures, as the static structure factor does not contain information about the temperature of the system. We are presently formulating a rigorous treatment of nonzero neutral temperatures using the dynamic structure factor [33].

All of our simulations were performed at different neutral densities as prescribed by the volume fraction $\phi$ and the hard-sphere radius of the single-particle cross section $\sigma_{\mathrm{sp}}$. The transport properties presented are independent of the neutral number density, as it scales inversely with the electric field strength. In all cases, the hard-sphere cross section for single-particle scattering was set as $\sigma_{\text {sp }}(\epsilon)=6 \AA^{2}$, while the charged particles were assigned a mass equal to that of an electron, $m=m_{e}$, and the mass of the neutrals was set to $m_{0}=4 u$, as used in previous studies [7]. While the techniques in this paper are readily applied to any energy-dependent anisotropic cross section set and any static structure factor, we present here only results for this analytically defined set of hard-sphere model systems, with the hope that it may serve as a simple, easily reproduced benchmark for future simulations and models.

\section{RESULTS AND DISCUSSION}

\section{A. Transport coefficients calculated with the proposed Monte Carlo method}

The Percus-Yevick hard-sphere system has been previously studied in [7], and a comparison with those results provides a test of our simulation [34]. Figure 4 shows the various transport coefficients simulated by the SSMC simulations, using the approach that overcomes the approximation of Wojcik and Tachiya. It is instructive to compare this figure with Fig. 3 to see the field strengths that are affected most strongly by the features of the structure factors employed. A table of some of our results is in Appendix B, where we have compared them with Boltzmann equation results. The complete set of all of our results is available as Supplementary Material [35]. We have simulated enough particles so that, in general, the Monte Carlo statistical error [36] is not visible at these scales, being less than $1 \%$ in all cases. Agreement with the Boltzmann equation results is to within $1 \%$ in all cases, so the datasets would not be seen as distinct if shown in the above figures, but a detailed comparison is presented in Sec. IV B.

The features of the results are discussed in detail in [7], and we will not repeat that discussion in depth here. One key feature is the presence of structure-induced negative-differential conductivity (defined in [37]) apparent in the drift velocity: at moderate field strengths of $1-10 \mathrm{Td}$, the drift velocity is inversely proportional to the field strength. This is because at low field strengths, the presence of coherent scattering causes anisotropy in particle scattering, which allows the particles 

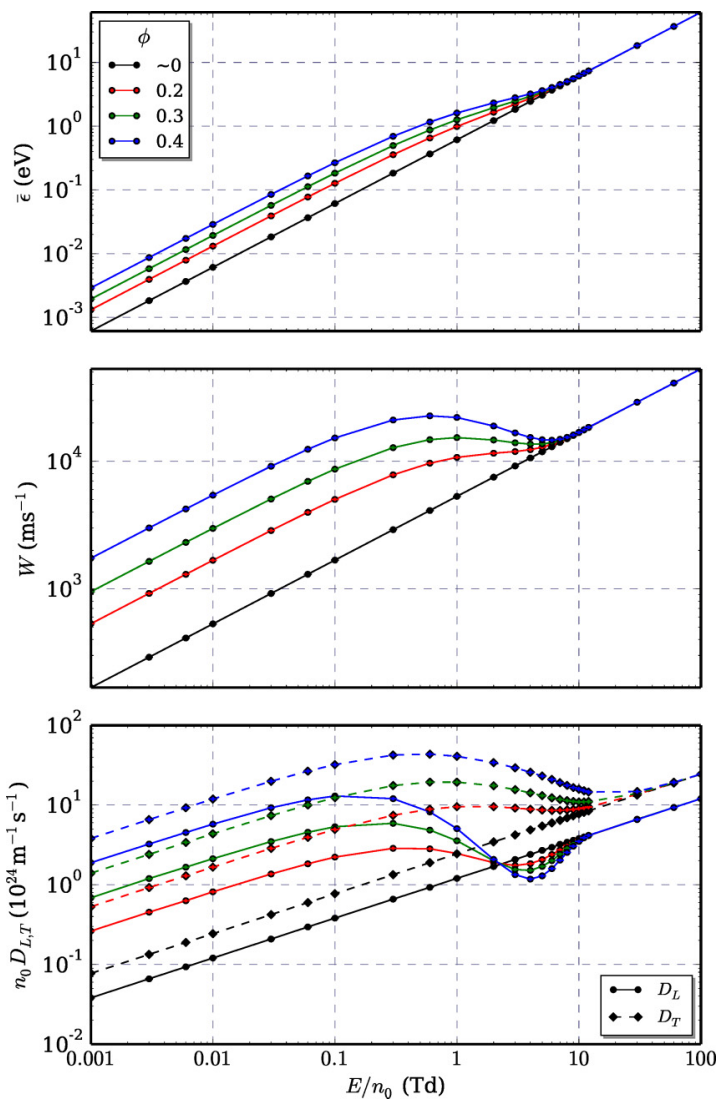

FIG. 4. (Color online) Mean energy $\epsilon$, drift velocity $W$, and diffusion coefficients $D_{L}$ and $D_{T}$ for Percus-Yevick model simulations, as a function of reduced electric field $E / n_{0}$ and Percus-Yevick packing ratio $\phi$. Error bars are not visible at this scale.

to be affected more consistently by the field, raising their velocity in comparison to the structure-free case. At higher field strengths, the mean particle energy is higher, leading to a reduced de Broglie wavelength, which means that the charged particles interact with fewer neutral molecules, so the coherent effects are reduced. This results in a net reduction of forward motion despite a higher average energy.

We would also like to highlight the variation in the anisotropic diffusion as a function of $\phi$. In the case of a hard-sphere gas with no structure, we expect the ratio $D_{L} / D_{T}=0.5$ [6], which, as shown in Fig. 5, is demonstrated by our simulations. When structure is introduced, this ratio changes significantly. This effect has been previously explored in [7] and [25] through the extended generalized Einstein relation. We note that a multiterm Boltzmann equation solution is required to achieve the accuracy of the SSMC technique. The SSMC simulations have no difficulties in accurately representing this anisotropy in the velocity distribution function.

\section{B. Comparisons with the Boltzmann equation and the method of Wojcik and Tachiya}

In Fig. 6, we present the percentage difference between our SSMC and WT results and the Boltzmann equation solution. Our implementation of the WT method shows good agreement over most regions of field strength; however, for some larger

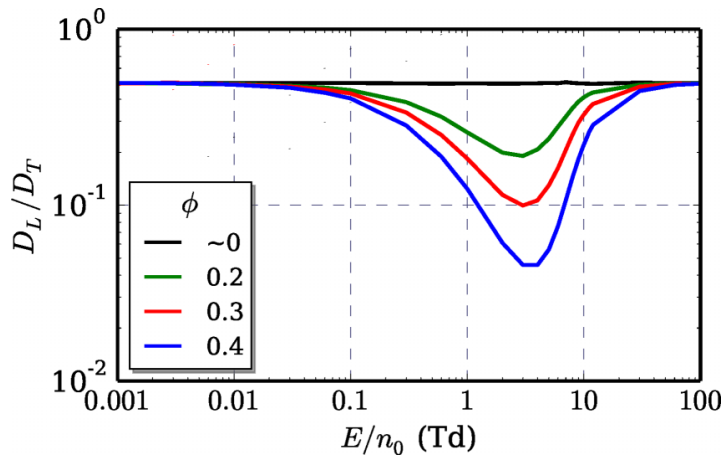

FIG. 5. (Color online) Ratio of diffusion coefficients as a function of reduced electric-field strength $E / n_{0}$ and packing ratio $\phi$. Note that the ratio for $\phi \sim 0$ is 0.5 , as required.

field strengths, errors of up to $5 \%$ in the mean energy and up to $35 \%$ in the diffusion coefficients become apparent. These differences occur when the energies of the electrons are within the regions that are truncated by the WT method.
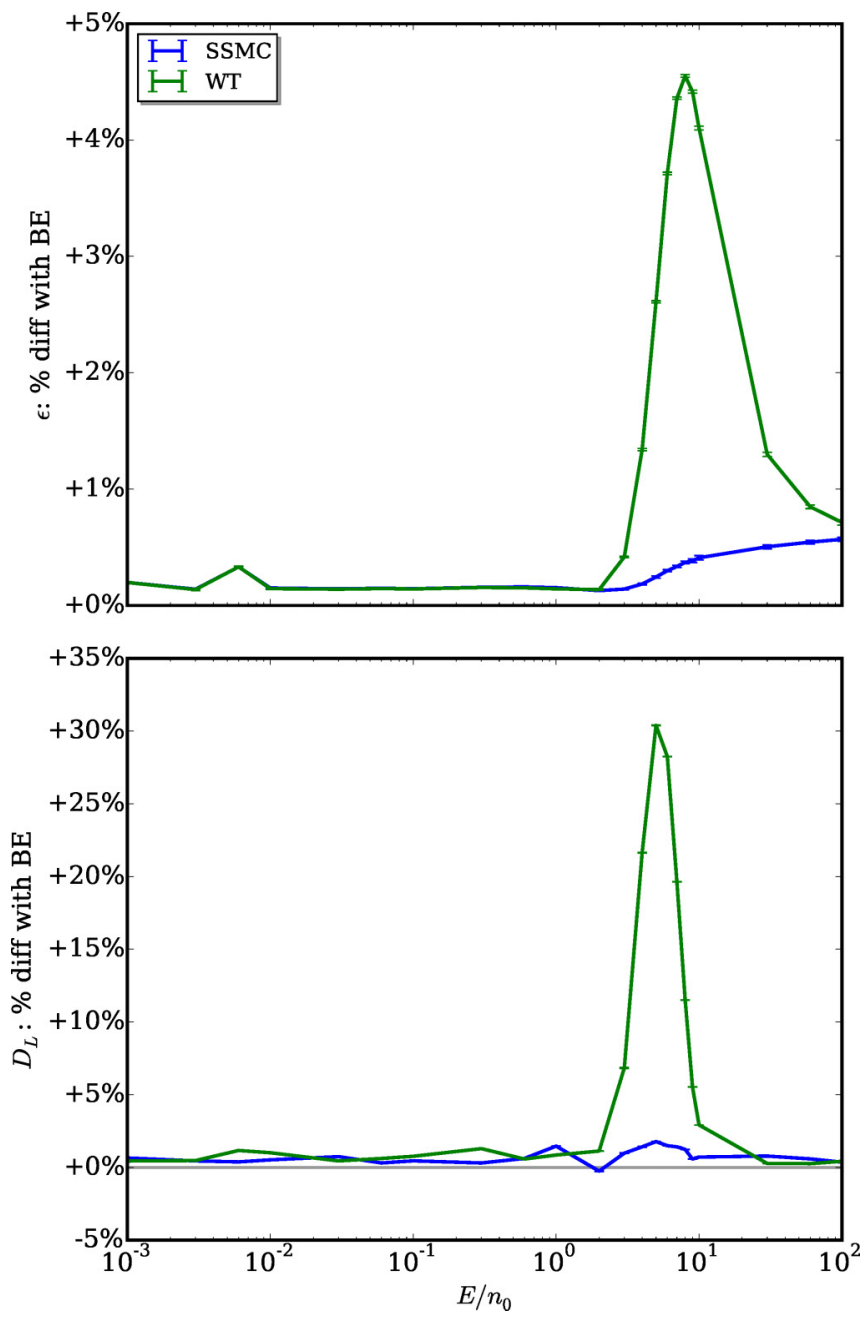

FIG. 6. (Color online) Mean energy $\epsilon$ and longitudinal diffusion $D_{L}$ percentage difference for each Monte Carlo model vs the Boltzmann equation (BE) model, for the Percus-Yevick structure factor at $\phi=0.4$. 
We can identify two competing factors that have an effect when the particle's energy is in these regions, causing differences between the SSMC and WT methods. First, the collision frequency is enhanced, and since every collision has a chance of both removing some energy from the particle and changing the direction of the particle away from the direction of the electric field, this means that particles will tend to lose energy at a greater rate. However, this is balanced by the presence of the momentum-only collision, which can occur up to $28 \%$ of the time in these regions. The presence of such collisions will tend to decrease the energy transfer rate. Nevertheless, Fig. 6 clearly shows that the combination of the effects is observable for the Percus-Yevick $\phi=0.4$ case, with a peak difference at approximately $8 \mathrm{Td}$. This corresponds to a mean energy of about $5 \mathrm{eV}$ (see Fig. 4), which is at the peak of the $\Gamma(\epsilon)$ function. That peak is where we would expect the WT approximation to be least suitable.

For the present model, the disagreements with the Boltzmann equation results are less than $1 \%$ over all field strengths considered. Such differences are of the order of the numerical schemes used in the SSMC and Boltzmann equation methods. We suspect the remaining differences are a result of energy meshes used in the Monte Carlo codes or Boltzmann equation numerical solutions.

\section{CONCLUDING REMARKS}

We have presented a Monte Carlo simulation code that accurately accounts for the effects of structure in nongaseous systems by employing a modified mechanistic per-collision interpretation of the Cohen and Lekner method for solving the Boltzmann equation. The SSMC results accurately replicate those calculated via a multiterm solution of the Boltzmann equation to within $1 \%$, with agreement significantly improved over those results obtained using the WT method, where errors of up to $35 \%$ were observed in some transport coefficients. Future work will be focused on utilizing a dynamic structure factor to account for other structural and collective effects, including nonzero background temperatures.

\section{APPENDIX A: COMPARISON BETWEEN MONTE CARLO AND BOLTZMANN EQUATION AVERAGES}

As discussed in Sec. II B, the three different processes in the SSMC have been chosen in order to reproduce the mean rate of transfer of energy and momentum that was obtained in the derivation of the Boltzmann equation. These different processes, corresponding to the "cross sections" defined in the main text, are as follows: $\sigma_{\text {both }}$, a regular single-neutral collision that exchanges both energy and momentum; $\sigma_{\text {energy }}$, a collision without direction change that aims to allow only energy exchange; and $\sigma_{\text {momentum, a collision with only }}$ momentum exchange. In this Appendix we show that, for isotropic scattering from stationary neutrals (i.e., we consider only $T=0$ in accordance with the regime of validity of the Monte Carlo simulations), these processes give rise to the same rates of energy and momentum transfer as the Boltzmann equation, after neglecting terms dependent on the mass ratio $m / m_{0}$. Although we describe these processes from the convenient viewpoint of single-neutral collisions, suitably modified to effect the desired change in momentum and energy, we emphasize that they are not intended to represent real collisions. Our goal is to reproduce the average rate of momentum and energy transfer from the electron to the medium as a whole, and so we are allowed to (and are in fact required to) deviate from the standard constraints of momentum and energy conservation for collision of an electron with a single neutral. Note that, in this Appendix, dashes refer to postcollision quantities.

In a collision, it is appropriate to consider the particle in the center-of-mass frame. In this frame, isotropic scattering implies that the particle's relative velocity $\boldsymbol{v}_{\text {rel }}=\boldsymbol{v}-\boldsymbol{g}$, where $\boldsymbol{g}$ is the velocity in the center-of-mass frame, is unchanged in magnitude $\left(\left|v_{\text {rel }}^{\prime}\right|=\left|v_{\text {rel }}\right|\right)$ and with a randomly assigned angle such that all values of $\cos \chi$, where $\chi$ is the scattering angle, are equally likely. We assume, without loss of generality, that the direction of the particle's velocity prior to the collision is aligned with the $z$ axis. Note that we do not need to consider the electric field in this discussion.

For a normal ("both") collision, one can easily show [6] that scattering from a stationary neutral leads to an angle-dependent energy change given by

$$
\Delta \epsilon_{\text {both }}=\epsilon \frac{2 m m_{0}}{\left(m+m_{0}\right)^{2}}(\cos \chi-1),
$$

and a change of the $z$ component of the momentum given by

$$
\Delta v_{z, \text { both }}=\frac{m_{0}}{m+m_{0}} v(\cos \chi-1) .
$$

Hence the average transfer of energy is

$$
\begin{aligned}
\langle\Delta \epsilon\rangle_{\text {both }} & =\frac{1}{2} \int_{-1}^{1} \Delta \epsilon_{\text {both }} d \cos \chi \\
& =2 m m_{0} \epsilon /\left(m+m_{0}\right)^{2}
\end{aligned}
$$

and the average transfer of momentum, for which the components perpendicular to $z$ average to zero, is

$$
\left\langle\Delta v_{z}\right\rangle_{\text {both }}=-m_{0} v_{z} /\left(m+m_{0}\right) .
$$

For an "energy-only" collision, we select a random change in energy through a random "false" angle $\chi$, and we calculate the energy change as if a normal collision through this angle had occurred. This preserves the average transfer of energy $\langle\Delta \epsilon\rangle_{\text {energy }}=\langle\Delta \epsilon\rangle_{\text {both }}$. We then discard the angle $\chi$, resetting the direction of motion of the particle to that before the collision. This has the unfortunate side effect of producing a change in momentum, contrary to the purpose of producing a collision with no momentum transfer. The change of momentum in this case is simply given by

$$
\begin{aligned}
\Delta v_{z, \text { energy }} & =\sqrt{\frac{2}{m}}(\sqrt{\epsilon-\Delta \epsilon(\chi)}-\sqrt{\epsilon}) \\
& =v\left(\sqrt{1+\frac{2 m m_{0}}{\left(m+m_{0}\right)^{2}}(\cos \chi-1)}-1\right),
\end{aligned}
$$


and upon averaging over $\cos \chi$, we find

$$
\begin{aligned}
\left\langle\Delta v_{z}\right\rangle_{\text {energy }} & =-v\left[1-\frac{1}{3 a}\left[1-(1-2 a)^{3 / 2}\right]\right] \\
& =-\frac{m\left(3 m_{0}-m\right) v_{z}}{3 m_{0}\left(m+m_{0}\right)}
\end{aligned}
$$

where $a=2 m m_{0} /\left(m+m_{0}\right)^{2}$, and the second result is arrived at after some algebraic manipulations, under the assumption that $m<m_{0}$.

For a "momentum-only" collision, we perform a collision as normal using an angle $\chi$ but set the postcollision energy to the precollision energy. Hence, it is obvious that $\langle\Delta \epsilon\rangle_{\text {momentum }}=0$, but the modification of the energy also has an impact on the average momentum change, which we calculate below. Note that $\boldsymbol{v}^{\prime}=v \hat{\boldsymbol{v}}^{\prime}$, where $\hat{\boldsymbol{v}}^{\prime}$ is identical to that of a normal collision. Hence,

$$
\boldsymbol{v}_{\text {both }}^{\prime} \cdot \hat{z}=\frac{v}{m+m_{0}}\left(m_{0} \cos \chi+m\right)
$$

implies that

$$
\begin{aligned}
\hat{\boldsymbol{v}}^{\prime} \cdot \hat{z} & =\frac{\boldsymbol{v}_{\text {both }}^{\prime} \cdot \hat{z}}{\left|\boldsymbol{v}_{\text {both }}^{\prime}\right|} \\
& =\frac{\cos \chi+\frac{m}{m_{0}}}{\sqrt{1+2 \frac{m}{m_{0}} \cos \chi+\frac{m^{2}}{m_{0}^{2}}}} .
\end{aligned}
$$

From this, we find the change in the $z$ component of momentum of a single collision,

$$
\Delta v_{z, \text { momentum }}=\left(\boldsymbol{v}^{\prime}-\boldsymbol{v}\right) \cdot \hat{z}=v\left(\hat{\boldsymbol{v}}^{\prime} \cdot \hat{z}-1\right),
$$

and hence we obtain an average momentum transfer of

$$
\begin{aligned}
\left\langle\Delta v_{z}\right\rangle_{\text {momentum }} & =\frac{v}{2} \int_{-1}^{1}\left(\frac{\cos \chi+\frac{m}{m_{0}}}{\sqrt{1+2 \frac{m}{m_{0}} \cos \chi+\frac{m^{2}}{m_{0}^{2}}}}-1\right) d \cos \chi \\
& =-v_{z}\left(1-\frac{2}{3} \frac{m}{m_{0}}\right) .
\end{aligned}
$$

We must now combine these averages. In the Monte Carlo simulation, there are two distinct regimes depending on the value of $\Gamma(\epsilon)$. If $\Gamma(\epsilon)<1$, then the $\sigma_{\text {both }}$ and $\sigma_{\text {energy }}$ cross sections occur with frequencies such that

$$
\begin{aligned}
\left.\frac{d\langle\epsilon\rangle}{d t}\right|_{\Gamma(\epsilon)<1} & =v_{\mathrm{sp}} \Gamma(\epsilon)\langle\Delta \epsilon\rangle_{\text {both }}+v_{\mathrm{sp}}[1-\Gamma(\epsilon)]\langle\Delta \epsilon\rangle_{\text {energy }} \\
& =v_{\mathrm{sp}}\langle\Delta \epsilon\rangle_{\text {both }} \\
& =v_{\mathrm{sp}} \frac{2 m m_{0}}{\left(m+m_{0}\right)^{2}} \epsilon,
\end{aligned}
$$

where $v_{\mathrm{sp}}(v)=n_{0} v \sigma_{\mathrm{sp}}(v)$ would be the collision frequency in the absence of structure effects, and

$$
\begin{aligned}
\left.m \frac{d\langle\boldsymbol{v}\rangle}{d t}\right|_{\Gamma(\epsilon)<1}= & m v_{\mathrm{sp}} \Gamma(\epsilon)\langle\Delta \boldsymbol{v}\rangle_{\text {both }} \\
& +m v_{\mathrm{sp}}[1-\Gamma(\epsilon)]\langle\Delta \boldsymbol{v}\rangle_{\text {energy }} \\
\approx & -\frac{m m_{0} v_{\mathrm{sp}} \boldsymbol{v}}{m+m_{0}} \Gamma(\epsilon)+O\left(\frac{m}{m_{0}}\right),
\end{aligned}
$$

where we have generalized the momentum transfer for any initial velocity direction, i.e., by replacing $v_{z}$ in Eqs. (A5) and (A10) by $\boldsymbol{v}$. To compare to the Boltzmann equations, we first note that $\sigma_{m}(v)=\sigma_{\mathrm{sp}}(v)$ for isotropic scattering and hence $v_{\mathrm{sp}}=v \Lambda_{0}^{-1}$ and $v_{\mathrm{sp}} \Gamma(\epsilon)=v \Lambda_{1}^{-1}$. Hence, we can see that these rates of transfer are in agreement with the results for the Boltzmann equations (3) and (4), except for some factors of order $m / m_{0}$ that are neglected in our simulations and in the derivation of the Boltzmann equation. If $\Gamma(\epsilon)>1$, then the "both" and "momentum-only" cross sections occur with frequencies such that

$$
\begin{aligned}
\left.\frac{d\langle\epsilon\rangle}{d t}\right|_{\Gamma(\epsilon)>1} & =v_{\mathrm{sp}}\langle\Delta \epsilon\rangle_{\mathrm{both}}+v_{\mathrm{sp}}[\Gamma(\epsilon)-1]\langle\Delta \epsilon\rangle_{\text {momentum }} \\
& =v_{\mathrm{sp}} \frac{2 m m_{0}}{\left(m+m_{0}\right)^{2}} \epsilon
\end{aligned}
$$

and

$$
\begin{aligned}
\left.m \frac{d\langle\boldsymbol{v}\rangle}{d t}\right|_{\Gamma(\epsilon)>1} & =m v_{\mathrm{sp}}\langle\Delta v\rangle_{\mathrm{both}}+m v_{\mathrm{sp}}[\Gamma(\epsilon)-1]\langle\Delta v\rangle_{\text {momentum }} \\
& \approx-\frac{m m_{0} v_{\mathrm{sp}} v}{m+m_{0}} \Gamma(\epsilon)+O\left(\frac{m}{m_{0}}\right),
\end{aligned}
$$

and again we recover the Boltzmann equation results with negligible factors of $\mathrm{m} / \mathrm{m}_{0}$.

We note that it is possible to correct for these differences of the order of $m / m_{0}$ by modifying the cross sections $\sigma_{\text {energy }}$ and $\sigma_{\text {momentum. }}$. However, this would only be necessary if we considered systems in which the mass ratios were close to unity.

Finally, we comment on the difference between the SSMC and WT methods. Wojcik and Tachiya [21] had mentioned that, in their particular calculation, they assumed the structure factor was mostly smaller than unity, which also implies that $\Gamma(\epsilon)<1$. They did not deal with values greater than unity, instead choosing to set $\Gamma(\epsilon)=\min (\Gamma(\epsilon), 1)$. This means that the rates of energy and momentum transfer are unchanged when $\Gamma(\epsilon)<1$ and continue to follow Eqs. (A11) and (A12). However, for $\Gamma(\epsilon)>1$, this leads to an error in the WT method in the momentum transfer, which is of the order

$$
-\frac{m m_{0} v_{\mathrm{sp}} v}{m+m_{0}}[\Gamma(\epsilon)-1] .
$$

There is no error in the energy transfer using the WT method.

\section{APPENDIX B: BENCHMARK VALUES FOR TRANSPORT COEFFICIENTS}

We present the benchmark values for the transport coefficients in Tables I and II. 
TABLE I. Benchmark values for transport coefficients: mean energy and drift velocity. Boltzmann equation (BE) results are compared with the SSMC results. The quoted error is twice the standard error in the mean of the SSMC results. Comparisons between BE and SSMC are shown as a third subcolumn for each type of transport coefficient.

\begin{tabular}{|c|c|c|c|c|c|c|c|}
\hline \multirow[b]{2}{*}{$\phi$} & \multirow{2}{*}{$\begin{array}{l}E / n_{0} \\
\text { (Td) }\end{array}$} & \multicolumn{3}{|c|}{ Mean energy $(\mathrm{eV})$} & \multicolumn{3}{|c|}{ Drift velocity $\left(\mathrm{ms}^{-1}\right)$} \\
\hline & & $\mathrm{BE}$ & SSMC & Diff. & $\mathrm{BE}$ & SSMC & Diff. \\
\hline \multirow[t]{5}{*}{0.0} & 0.03 & 0.018232 & $0.018338 \pm 0.023 \%$ & $0.58 \%$ & 910.63 & $918.52 \pm 0.0058 \%$ & $0.86 \%$ \\
\hline & 0.3 & 0.18232 & $0.1834 \pm 0.022 \%$ & $0.59 \%$ & 2879.7 & $2905.6 \pm 0.0064 \%$ & $0.90 \%$ \\
\hline & 3 & 1.8232 & $1.8333 \pm 0.02 \%$ & $0.55 \%$ & 9106.3 & $9186.5 \pm 0.0074 \%$ & $0.88 \%$ \\
\hline & 10 & 6.077 & $6.113 \pm 0.022 \%$ & $0.58 \%$ & 16626 & $16783 \pm 0.0096 \%$ & $0.94 \%$ \\
\hline & 30 & 18.232 & $18.34 \pm 0.024 \%$ & $0.58 \%$ & 28797 & $29077 \pm 0.01 \%$ & $0.97 \%$ \\
\hline \multirow[t]{5}{*}{0.2} & 0.03 & 0.039039 & $0.039149 \pm 0.012 \%$ & $0.28 \%$ & 2851.35 & $2861.91 \pm 0.002 \%$ & $0.37 \%$ \\
\hline & 0.3 & 0.35569 & $0.35667 \pm 0.015 \%$ & $0.27 \%$ & 7797 & $7827.7 \pm 0.0027 \%$ & $0.39 \%$ \\
\hline & 3 & 2.2039 & $2.2102 \pm 0.014 \%$ & $0.28 \%$ & 11860.7 & $11921.1 \pm 0.0066 \%$ & $0.50 \%$ \\
\hline & 10 & 6.112 & $6.141 \pm 0.017 \%$ & $0.48 \%$ & 16726 & $16851.5 \pm 0.0055 \%$ & $0.75 \%$ \\
\hline & 30 & 18.25 & $18.351 \pm 0.023 \%$ & $0.55 \%$ & 28833 & $29071 \pm 0.0048 \%$ & $0.82 \%$ \\
\hline \multirow[t]{5}{*}{0.3} & 0.03 & 0.057118 & $0.057228 \pm 0.01 \%$ & $0.19 \%$ & 5044.64 & $5055.53 \pm 0.0011 \%$ & $0.21 \%$ \\
\hline & 0.3 & 0.49489 & $0.49585 \pm 0.01 \%$ & $0.19 \%$ & 12756.1 & $12788.1 \pm 0.0016 \%$ & $0.25 \%$ \\
\hline & 3 & 2.4654 & $2.4703 \pm 0.012 \%$ & $0.20 \%$ & 13909 & $13959.3 \pm 0.0047 \%$ & $0.36 \%$ \\
\hline & 10 & 6.127 & $6.152 \pm 0.017 \%$ & $0.41 \%$ & 16756.5 & $16863.1 \pm 0.004 \%$ & $0.63 \%$ \\
\hline & 30 & 18.259 & $18.354 \pm 0.015 \%$ & $0.51 \%$ & 28852.2 & $29065.3 \pm 0.0031 \%$ & $0.73 \%$ \\
\hline \multirow{5}{*}{0.4} & 0.03 & 0.084858 & $0.084979 \pm 0.0098 \%$ & $0.14 \%$ & 9132.66 & $9139.8 \pm 0.00079 \%$ & $0.08 \%$ \\
\hline & 0.3 & 0.69229 & $0.69337 \pm 0.0083 \%$ & $0.15 \%$ & 21031.8 & $21066.9 \pm 0.0012 \%$ & $0.16 \%$ \\
\hline & 3 & 2.7924 & $2.7963 \pm 0.0065 \%$ & $0.14 \%$ & 16637.7 & $16680.3 \pm 0.0038 \%$ & $0.25 \%$ \\
\hline & 10 & 6.14 & $6.165 \pm 0.018 \%$ & $0.40 \%$ & 16767.6 & $16871.6 \pm 0.0033 \%$ & $0.62 \%$ \\
\hline & 30 & 18.269 & $18.361 \pm 0.017 \%$ & $0.50 \%$ & 28872.5 & $29076.6 \pm 0.0028 \%$ & $0.70 \%$ \\
\hline
\end{tabular}

TABLE II. Benchmark values for transport coefficients: longitudinal and transverse diffusion, and energy gradient parameter. Boltzmann equation (BE) results are compared with the SSMC results. The quoted error is twice the standard error in the mean of the SSMC results. Comparisons between BE and SSMC are shown as a third subcolumn for each type of transport coefficient.

\begin{tabular}{|c|c|c|c|c|c|c|c|}
\hline \multirow[b]{2}{*}{$\phi$} & \multirow{2}{*}{$\begin{array}{l}E / n_{0} \\
(\mathrm{Td})\end{array}$} & \multicolumn{3}{|c|}{$n_{0} D_{L}\left(10^{24} \mathrm{~m}^{-1} \mathrm{~s}^{-1}\right)$} & \multicolumn{3}{|c|}{$n_{0} D_{T}\left(10^{24} \mathrm{~m}^{-1} \mathrm{~s}^{-1}\right)$} \\
\hline & & $\mathrm{BE}$ & SSMC & Diff. & $\mathrm{BE}$ & SSMC & Diff. \\
\hline \multirow[t]{5}{*}{0.0} & 0.03 & 0.20726 & $0.20859 \pm 0.01 \%$ & $0.64 \%$ & 0.42195 & $0.4224 \pm 0.0041 \%$ & $0.10 \%$ \\
\hline & 0.3 & 0.65543 & $0.66011 \pm 0.011 \%$ & $0.71 \%$ & 1.33432 & $1.33984 \pm 0.006 \%$ & $0.41 \%$ \\
\hline & 3 & 2.0726 & $2.0767 \pm 0.014 \%$ & $0.19 \%$ & 4.2195 & $4.2261 \pm 0.0059 \%$ & $0.15 \%$ \\
\hline & 10 & 3.7841 & $3.8073 \pm 0.012 \%$ & $0.61 \%$ & 7.7037 & $7.7459 \pm 0.0067 \%$ & $0.54 \%$ \\
\hline & 30 & 6.5543 & $6.6398 \pm 0.012 \%$ & $1.30 \%$ & 13.3432 & $13.3487 \pm 0.0059 \%$ & $0.04 \%$ \\
\hline \multirow[t]{5}{*}{0.2} & 0.03 & 1.35966 & $1.36532 \pm 0.006 \%$ & $0.41 \%$ & 2.8418 & $2.8603 \pm 0.0091 \%$ & $0.65 \%$ \\
\hline & 0.3 & 2.8614 & $2.8671 \pm 0.0092 \%$ & $0.19 \%$ & 7.4604 & $7.4461 \pm 0.0032 \%$ & $-0.19 \%$ \\
\hline & 3 & 1.7277 & $1.7446 \pm 0.022 \%$ & $0.97 \%$ & 9.1902 & $9.1665 \pm 0.0066 \%$ & $-0.25 \%$ \\
\hline & 10 & 3.677 & $3.7075 \pm 0.018 \%$ & $0.82 \%$ & 9.0054 & $9.0232 \pm 0.0048 \%$ & $0.19 \%$ \\
\hline & 30 & 6.5411 & $6.5948 \pm 0.012 \%$ & $0.82 \%$ & 13.6167 & $13.6324 \pm 0.0042 \%$ & $0.11 \%$ \\
\hline \multirow[t]{5}{*}{0.3} & 0.03 & 3.4743 & $3.4834 \pm 0.0071 \%$ & $0.26 \%$ & 7.3668 & $7.3715 \pm 0.0086 \%$ & $0.06 \%$ \\
\hline & 0.3 & 5.8751 & $5.9043 \pm 0.0076 \%$ & $0.49 \%$ & 17.561 & $17.571 \pm 0.0048 \%$ & $0.06 \%$ \\
\hline & 3 & 1.5321 & $1.5553 \pm 0.018 \%$ & $1.50 \%$ & 15.651 & $15.626 \pm 0.0074 \%$ & $-0.15 \%$ \\
\hline & 10 & 3.5985 & $3.5869 \pm 0.023 \%$ & $-0.32 \%$ & 11.029 & $10.952 \pm 0.0096 \%$ & $-0.70 \%$ \\
\hline & 30 & 6.5336 & $6.5951 \pm 0.0065 \%$ & $0.94 \%$ & 13.985 & $13.991 \pm 0.0072 \%$ & $0.04 \%$ \\
\hline \multirow[t]{5}{*}{0.4} & 0.03 & 9.182 & $9.2498 \pm 0.0041 \%$ & $0.73 \%$ & 19.8166 & $19.8841 \pm 0.004 \%$ & $0.34 \%$ \\
\hline & 0.3 & 11.9922 & $12.0287 \pm 0.0071 \%$ & $0.30 \%$ & 42.211 & $42.43 \pm 0.0069 \%$ & $0.51 \%$ \\
\hline & 3 & 1.3271 & $1.34 \pm 0.027 \%$ & $0.97 \%$ & 29.2923 & $29.3425 \pm 0.0017 \%$ & $0.17 \%$ \\
\hline & 10 & 3.4836 & $3.5084 \pm 0.0072 \%$ & $0.71 \%$ & 15.81 & $15.813 \pm 0.0067 \%$ & $0.02 \%$ \\
\hline & 30 & 6.526 & $6.5769 \pm 0.0062 \%$ & $0.77 \%$ & 14.7608 & $14.7684 \pm 0.0038 \%$ & $0.05 \%$ \\
\hline
\end{tabular}


[1] D. Carlson and C. Wronski, in Amorphous Semiconductors, 2nd ed., edited by M. H. Brodsky (Springer-Verlag, Berlin, Heidelberg, 1985), pp. 113-158.

[2] H. Nikjoo, M. Terrissol, R. Hamm, J. Turner, S. Uehara, H. Paretzke, and D. Goodhead, Radiat. Protect. Dosimetry 52, 165 (1994).

[3] D. W. Gidley, H.-G. Peng, and R. S. Vallery, Annu. Rev. Mater. Res. 36, 49 (2006)

[4] I. Buvat, I. Castiglioni, J. Feuardent, and M. C. Gilardi, Phys. Med. Biol. 50, 329 (2005).

[5] I. Kawrakow and A. Bielajew, Nucl. Instrum. Methods Phys. Res. B 142, 253 (1998).

[6] R. E. Robson, Introductory Transport Theory for Charged Particles in Gases (World Scientific, Singapore, 2006).

[7] R. D. White and R. E. Robson, Phys. Rev. E 84, 031125 (2011).

[8] A. O. Allen, P. J. Kuntz, and W. F. Schmidt, J. Phys. Chem. 88, 3718 (1984).

[9] C. Champion, C. Le Loirec, and B. Stosic, Int. J. Radiat. Biol. 88, 54 (2012).

[10] D. Emfietzoglou, K. Karava, G. Papamichael, and M. Moscovitch, Phys. Med. Biol. 48, 2355 (2003).

[11] M. Šuvakov, Z. L. Petrović, J. P. Marler, S. J. Buckman, R. E. Robson, and G. Malović, New J. Phys. 10, 053034 (2008).

[12] A. Muñoz, J. M. Pérez, G. García, and F. Blanco, Nucl. Instrum. Methods Phys. Res. Sect. A 536, 176 (2005).

[13] H. R. Skullerud, J. Phys. D 1, 1567 (1968).

[14] Y. Sakai, K. Sukegawa, S. Nakamura, and H. Tagashira, IEEE Trans. Elect. Insulat. 23, 609 (1988).

[15] C. Champion and C. Le Loirec, Phys. Med. Biol. 51, 1707 (2006).

[16] K. Huthmacher, A. Herzwurm, M. Gnewuch, K. Ritter, and B. Rethfeld, Physica A 429, 242 (2015).

[17] P. Verkerk, U. Bafile, F. Barocchi, L. A. de Graaf, J.-B. Suck, and H. Mutka, Phys. Rev. Lett. 67, 1262 (1991).

[18] M. Michaud, A. Wen, and L. Sanche, Radiat. Res. 159, 3 (2003).

[19] J.-C. Kuhr and H.-J. Fitting, J. Electron Spectrosc. Relat. Phenom. 105, 257 (1999).

[20] A. Muñoz, F. Blanco, G. Garcia, P. Thorn, M. Brunger, J. Sullivan, and S. Buckman, Int. J. Mass Spectrom. 277, 175 (2008).
[21] M. Wojcik and M. Tachiya, Chem. Phys. Lett. 363, 381 (2002).

[22] L. Verlet and J.-J. Weis, Phys. Rev. A 5, 939 (1972).

[23] M. Cohen and J. Lekner, Phys. Rev. 158, 305 (1967).

[24] L. Van Hove, Phys. Rev. 95, 249 (1954).

[25] G. J. Boyle, R. D. White, R. E. Robson, S. Dujko, and Z. L. Petrović, New J. Phys. 14, 045011 (2012).

[26] Note that single-particle cross sections are ideally defined in terms of the collision energy, which depends on the relative speed of the particle in the center-of-mass frame of the collision. However, in practice, most measured cross sections are, by necessity, the integral of the cross section of all possible relative speeds, weighted by their frequency according to the neutral particle's velocity distribution. The differences are very small for electrons impacting on even the lightest atoms, although they may be significant for ion scattering at very low energies.

[27] M. J. Brennan, A. M. Garvie, and L. J. Kelly, Aust. J. Phys. 43, 27 (1990).

[28] M. J. Brennan, IEEE Trans. Plasma Sci. 19, 256 (1991).

[29] M. Wertheim, Phys. Rev. Lett. 10, 321 (1963).

[30] E. Thiele, J. Chem. Phys. 39, 474 (1963).

[31] A. E. Nahum, Radiat. Environ. Biophys. 38, 163 (1999).

[32] S. Dujko, R. D. White, and Z. L. Petrović, J. Phys. D 41, 245205 (2008).

[33] W. J. Tattersall, D. G. Cocks, and R. D. White (unpublished).

[34] An error in the calculation of the Percus-Yevick structure factor used in [7] means that the structure factor would be correct for a fluid where the molecules have a hard-sphere cross section of $\sigma=1.5 \AA^{2}$, not the reported cross section of $\sigma=6 \AA^{2}$, although the cross sections themselves were as reported. The Boltzmann equation results presented here have been recalculated with the correct structure factor, as described in Sec. III A. The phenomenology reported in [7] remains correct, however.

[35] See Supplemental Material at http://link.aps.org/supplemental/ 10.1103/PhysRevE.91.043304 for the complete set of transport coefficients at all simulated reduced electric-field strengths.

[36] E. Koehler, Am. Statist. 63, 155 (2009).

[37] R. D. White and R. E. Robson, Phys. Rev. Lett. 102, 230602 (2009). 